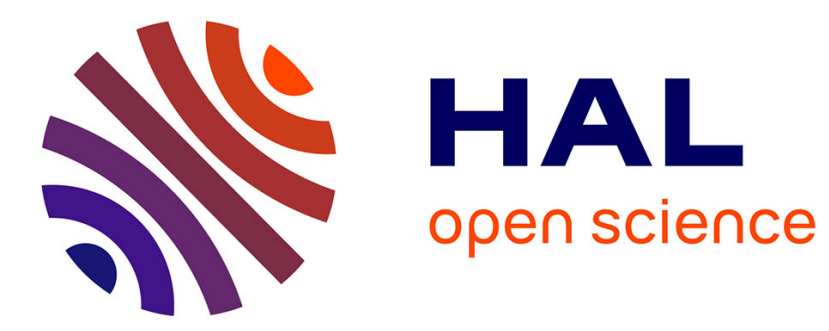

\title{
Waveguiding in blazed-binary diffractive elements
}

Philippe Lalanne

\section{To cite this version:}

Philippe Lalanne. Waveguiding in blazed-binary diffractive elements. Journal of the Optical Society of America. A Optics, Image Science, and Vision, 1999, 16 (10), pp.2517-2520. hal-00867723

\section{HAL Id: hal-00867723 \\ https://hal-iogs.archives-ouvertes.fr/hal-00867723}

Submitted on 30 Sep 2013

HAL is a multi-disciplinary open access archive for the deposit and dissemination of scientific research documents, whether they are published or not. The documents may come from teaching and research institutions in France or abroad, or from public or private research centers.
L'archive ouverte pluridisciplinaire HAL, est destinée au dépôt et à la diffusion de documents scientifiques de niveau recherche, publiés ou non, émanant des établissements d'enseignement et de recherche français ou étrangers, des laboratoires publics ou privés. 


\title{
Waveguiding in blazed-binary diffractive elements
}

\author{
Philippe Lalanne \\ Laboratoire Charles Fabry de l'Institut d'Optique, Centre National de la Recherche Scientifique, \\ B.P. 147, F-91403 Orsay Cedex, France
}

Received December 23, 1998; accepted May 25, 1999

\begin{abstract}
Recent experimental and numerical results clearly show that blazed-binary diffractive elements outperform their standard blazed-échelette counterparts in the resonance domain. A theoretical study of one-dimensional blazed-binary gratings shows that the reason for this high efficiency is a waveguiding effect. The electromagnetic study supports the idea that, through waveguiding, a reduction of the shadowing zone is achieved, and thus the efficiency is increased. This is intrinsic to high-frequency binary structures and cannot be achieved with standard échelette diffractive elements. (c) 1999 Optical Society of America [S0740-3232(99)00410-X] OCIS codes: 050.1380, 050.1970, 050.1950.
\end{abstract}

Blazed-binary optical elements [see Fig. 1(c)] are diffractive components with binary profiles that achieve a high diffraction efficiency of a specified order. They are composed of subwavelength ridges, pillars, or other simple geometries carefully etched into a dielectric film. In general the subwavelength features are arranged on a regular grid. The periodicity of the grid is called the sampling period because the phase of the diffractive element is sampled at the grid point locations. For ridges or low-symmetry features such as rectangles, a form of birefringence is induced, and the diffractive component is highly sensitive to the polarization of the incident radiation. Conversely, when centrosymmetric subwavelength features such as pillars or cylinders are involved, the diffractive elements can be operated with unpolarized light. The design of blazed-binary optical elements strongly relies on the analogy between subwavelength gratings and homogeneous thin films. In general terms, the incident wave averages the subwavelength features, and an artificial medium is generated. ${ }^{1}$ The local effective index experienced by the incident wave is related to the local fraction of etched material. ${ }^{2}$ Consequently, when the size of the subwavelength features is controlled, arbitrary refractive-index distributions can be synthesized on a substrate, and blazing with binary features is thus achieved.

Recently, blazed-binary optical elements with diffraction efficiencies significantly higher than those of their corresponding blazed-échelette [see Fig. 1(a)] counterparts were fabricated. ${ }^{3,4}$ Trying to explain this enhanced performance, the authors of Ref. 4 referred to a reduction of the shadowing zone and to a surprising sampling effect. Although the former has only a small effect on the performance enhancement, numerical results showed that the performance of blazed-binary gratings strongly depends on the choice of the sampling period. Contrary to the intuition gained from the equivalence between subwavelength gratings and homogeneous thin films in the static limit (for sampling periods much smaller than the wavelength), much better diffraction performance is achieved for sampling-period values barely smaller than the structural-cutoff period, above which the anal ogy between subwavelength gratings and homogeneous thin layer ceases to be valid. The physical reason for this surprising effect was not given in Ref. 4.

In this paper I interpret the origin of the enhanced performance as the effect of waveguiding through the diffractive el ement. This interpretation is supported by plots of the electromagnetic field distribution in a very simple structure: a blazed-binary grating with one-dimensional subwavelength ridges and grooves. Although this study is restricted to gratings with one-dimensional subwavelength geometries, I believe that the interpretation also holds for diffractive elements composed of twodimensional subwavelength features as in Ref. 4.

In what follows, a normally incident plane wave (wavelength in vacuum $\lambda$ ) from a glass substrate (refractive index $n_{g}=1.52$ ) is assumed. The numerical study is performed for TM polarization (magnetic field vector parallel to the ridges). Similar results (not reported here) were obtained for gratings operating under TE polarization (electric field vector parallel to the ridges). The grating period $\Lambda$ is equal to $3 \lambda$. In view of the results of Ref. 4, several sampling periods are considered. The blazedbinary gratings are designed to mimic a blazed-index grating [see Fig. 1(b)] with a refractive index linearly and continuously varying from 1 to a maximum value $\mathrm{n}_{\max }$ $=1.67$ within the period. The blazed-index case is simply the ideal structure that one attempts to mi mick when considering blazed-binary diffractive elements for fabrication purposes. The blazed-binary gratings are assumed to be etched in $\mathrm{TiO}_{2}$, refractive index 2.3. The designs strictly follow procedure 2 of Ref. 4: After a calibration curve relating the effective index to the ridge width is computed, the widths of the subwavelength ridges are fixed by each sampling location's being assigned the corresponding effective index, with the grating depth assumed to be equal to $\lambda /\left(n_{\max }-1\right)$. In this theoretical study we allow for the fabrication of arbitrarily small ridges and grooves.

In the first step, let us focus on the far-field performance. Table 1 shows the first-order diffraction effi- 


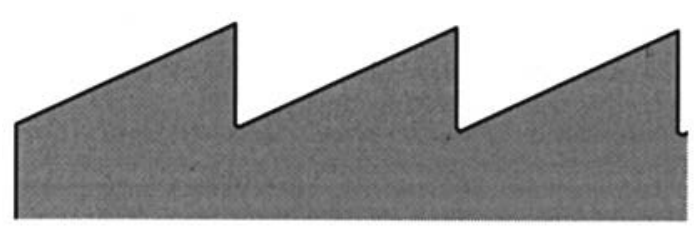

(a)

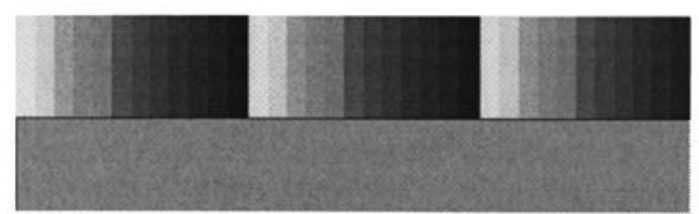

(b)

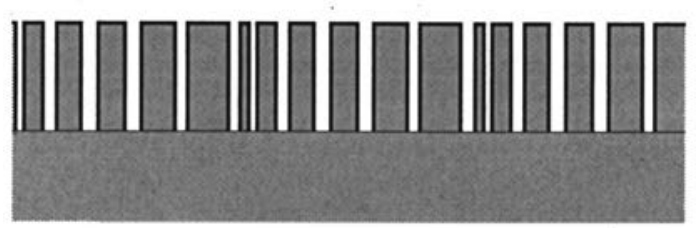

(c)

Fig. 1. (a) Blazed-échelette, (b) blazed-index, (c) blazed-binary gratings.

\section{Table 1. First-Order Diffraction Efficiency

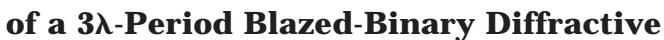 Grating as a Function of the Number (N) of Subwavelength Ridges ${ }^{a}$}

\begin{tabular}{rc}
\hline$N$ & Efficiency (\%) \\
\hline 3 & 67.2 \\
4 & 88.7 \\
5 & 86.3 \\
6 & 85.3 \\
7 & 87.1 \\
8 & 77.8 \\
9 & 75.9 \\
10 & 76.0 \\
\hline
\end{tabular}

${ }^{a}$ Results hold for TM polarization under normal incidence from the glass substrate.

ciency for different values of $\mathrm{N}$, the number of ridges per period. The numerical results are obtained by use of the enhanced version ${ }^{5}$ of rigorous coupled-wave analysis. ${ }^{6}$ The enhanced version is a versatile and efficient tool for modeling the diffraction of electromagnetic waves by onedimensional periodic structures under TM polarization and conical mountings. Table 1 reveals that, as was observed for two-dimensional subwavelength features, ${ }^{4}$ the performance strongly depends on the sampling period, with a maximum for relatively large sampling periods.

To gain physical insight into this sampling effect, let us now examine the near-field electromagnetic response. Figures 2(b)-2(f) show the magnetic field distribution inside several blazed-binary gratings for $\mathrm{N}=4,5,6,8,10$. For comparison, the field distribution for the corresponding blazed-index grating is plotted in Fig. 2(a). The white arrows in the two upper plots represent the Poynting vectors. These are not shown in Figs. 2(c)-2(f) for the sake of clarity. The field distributions and Poynting vectors in Fig. 2 are obtained by use of the numerical method described in Ref. 7. This method, based on rigorous coupled-wave analysis, is highly accurate: Even the field singularities near wedges are correctly estimated, for instance. The magnitude of the incident magnetic field is unity.

Qualitatively, Fig. 2 shows that the magnetic field is larger in the subwavelength ridges than in the grooves. This is especially extreme for the few larger ridges in Figs. 2(b)-2(e). Moreover, the Poynting vector map in Fig. 2(b) indicates that the energy flow inside the grating region is confined within the ridges. This effect is, of course, not present for the blazed-index case of Fig. 2(a). Qualitatively, the ridges act as waveguides. This interpretation can be made more quantitative. A careful inspection of Figs. 2(b)-2(e) reveals the presence of bright spots or antinodes for the magnetic fields inside the few larger ridges. These bright spots result from standing waves produced by the reflections of the light stream experienced by the ridges at the upper and lower grating interfaces. The distance between two successive antinodes $\mathrm{i}$ is related to the effective index $\mathrm{n}$ of the light stream, $\mathrm{i}$ $=\lambda /(2 \mathrm{n})$. For the two largest ridges in Fig. 2(b), the effective indices measured on the plot agree within a few percent with those obtained by numerical computation for the fundamental mode of single isolated slab waveguides with identical core widths and with identical index profiles ( 2.3 for the core and 1 for the substrate and superstrate). A less accurate agreement (15\%) is obtained for the next-largest ridge in Fig. 2(b). This is probably because, for this narrow-ridge case, the fundamental mode of the slab waveguide spreads far away from its core region and overlaps with the neighboring grating ridges. In other words, the isolated waveguide model is no longer valid. For decreasing sampling periods [Figs. 2(c)-2(e)] the interaction between adjacent ridges grows even stronger, and the isolated waveguide model becomes less and less valid.

The diffraction efficiency of the blazed-index grating in Fig. 2(a) is $72 \%$, a value $16 \%$ smaller than that achieved with the four-ridge grating considered in Fig. 2(b). A comparative inspection of the Poynting vectors is instructive for understanding why. First, note that the energy flow for the blazed-index grating is strongly disturbed, especially in the high-index region near the discontinuity [right-hand side in Fig. 2(a)]. Intuitively, this perturbation can be interpreted as the shadowing effect or, equivalently, as the total internal reflection appearing at the grating discontinuity; see Ref. 4. In contrast, a relatively uniform energy flow is observed for the blazed-binary case of Fig. 2(b). As a consequence of the waveguiding effect of the subwavelength ridges, an intense and coherent wave is able to flow straight through the grating, and a high diffraction efficiency is achieved. In other words, this waveguiding effect may be seen as responsible for an effective reduction of the shadowing zone. This property is intrinsic to diffractive elements composed of subwavelength features and cannot be achieved with standard blazed-index or blazed-échel ette diffractive elements. 
(a)

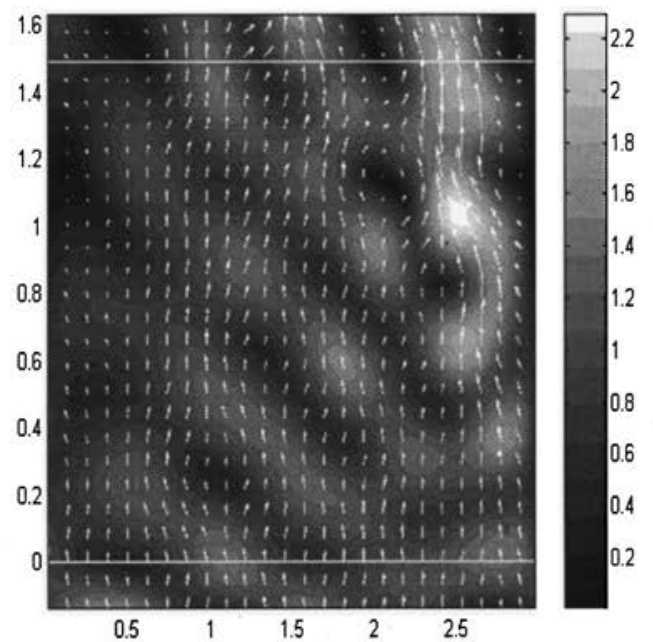

(c)

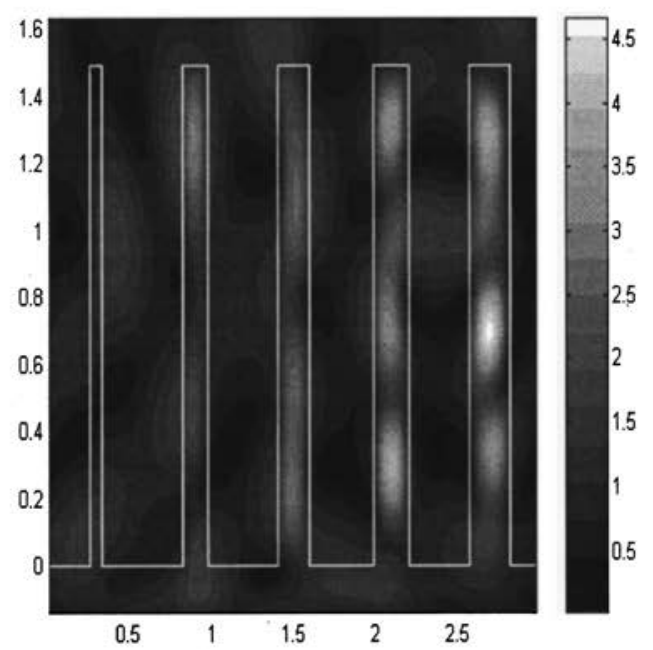

(e)

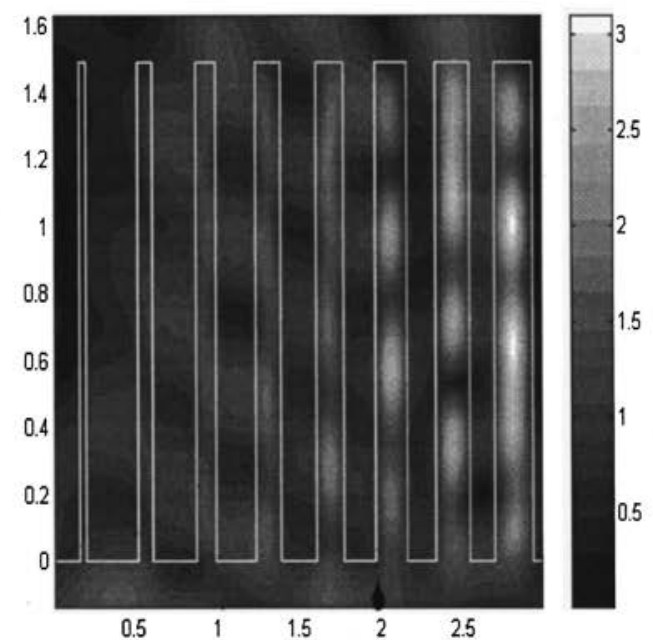

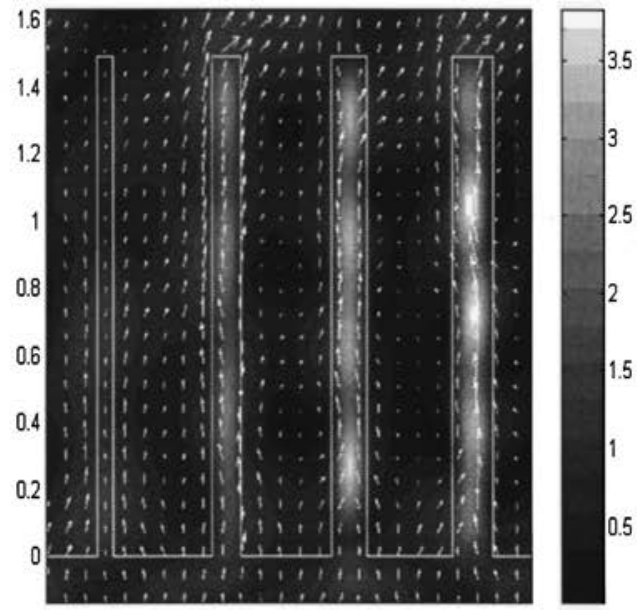

(b)

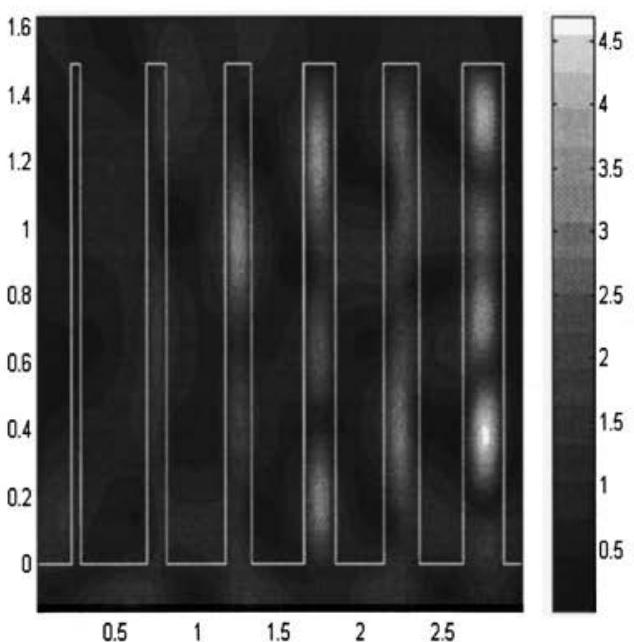

(d)

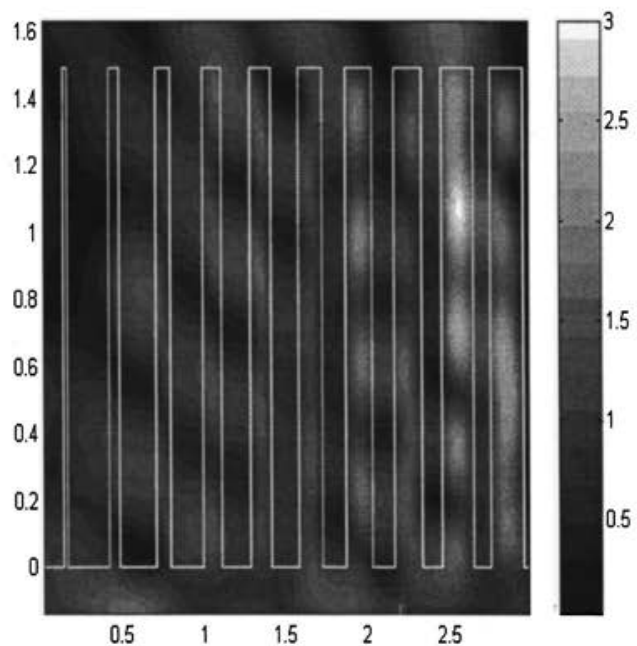

(f)

Fig. 2. Distribution of the magnitude of the magnetic field vector inside several blazed gratings. (a) Blazed-index grating. In this case the grating refractive index varies linearly between 1 and $n_{\max }$ within one period. The horizontal white lines delimitate the grating region. The first-order diffraction efficiency is $72 \%$. (b)-(f) Blazed-binary gratings with $\mathrm{N}=4,5,6,8,10$. The white arrows in (a) and (b) represent the Poynting vectors. For all plots the vertical $z$ and horizontal $x$ axes are the same. The gratings are all illuminated from the glass substrate (half-plane $z<0$ ), and along the $x$ axis one grating period is shown. The interface between the gratings and the substrate (air) is the plane $z=\lambda /\left(\mathrm{n}_{\max }-1\right)$. 
The author may be reached at philippe.lalanne@iota. u-psud.fr.

\section{REFERENCES}

1. D. E. Aspnes, "Local-field effects and effective-medium theory: a microscopic perspective," Am. J . Phys. 50, 704 709 (1982).

2. W. Stork, N. Streibl, H. Haidner, and P. Kipfer, "Artificial distributed-index media fabricated by zero-order gratings," Opt. Lett. 16, 1921-1923 (1991).

3. Ph. Lalanne, S. Astilean, P. Chavel, E. Cambril, and H. Launois, "Blazed-binary subwavelength gratings with effi- ciencies larger than those of conventional échelette gratings," Opt. Lett. 23, 1081-1083 (1998).

4. Ph. Lalanne, S. Astilean, P. Chavel, E. Cambril, and H. Launois, "Design and fabrication of blazed-binary diffractive el ements with sampling periods smaller than the structural cutoff," J . Opt. Soc. Am. A 16, 1143-1156 (1999).

5. Ph. Lalanne and G. M. Morris, "Highly improved convergence of the coupled-wave method for TM polarization,"J . Opt. Soc. Am. A 13, 779-784 (1996).

6. M. G. Moharam, E. B. Grann, D. A. Pommet, and T. K. GayIord, "Formulation for stable and efficient implementation of the rigorous coupled-wave analysis of binary gratings,"J . Opt. Soc. Am. A 12, 1068-1076 (1995).

7. Ph. Lalanne and M. P. J urek, "Computation of the nearfield pattern with the coupled-wave method for TM polarization," J . Mod. Opt. 45, 1357-1374 (1998). 